\title{
ITERATIVE METHOD TO FIND CORE-EP INVERSE
}

\author{
Manjunatha Prasad Karantha*, David Raj Micheal ${ }^{* * 1}$, M. Vinay ${ }^{* * *}$ \\ *Department of Statistics, PSPH, Manipal Academy of Higher Education, \\ Manipal 576104, India \\ kmprasad63@gmail.com, km.prasademanipal.edu \\ ** Department of Statistics, PSPH, Manipal Academy of Higher Education, \\ Manipal 576104, India \\ daviddgl20130gmail.com \\ ${ }^{* * *}$ Department of Mathematics, Manipal Institute of Technology, MAHE, \\ Manipal 576104, India \\ vinay.m2000@gmail.com
}

(Received 20.01.2018, accepted 19.03.2018

\begin{abstract}
In this article, an iterative method for estimating the core-EP inverse is proposed and the convergence properties of the same has been discussed. Also, numerical examples with different values of parameters and the criteria for stopping the iteration are presented. classification: $15 \mathrm{~A} 09 ; 65 \mathrm{~F} 10$

keywords: generalized inverse; outer inverse; EP matrix; core-inverse; core-EP inverse; iterative method
\end{abstract}

\section{Introduction}

Most frequently used recursion relation for finding the inverse $A^{-1}$ of a matrix $A$ is $V_{k+1}=V_{k}+V_{k}\left(I-A V_{k}\right)$, which is known to be Newton's recursive formula. Schulz in [15] proved that the recursive matrix in the Newton's iteration converges if

$$
\rho\left(I-A V_{0}\right)<1,
$$

1 Corresponding author 
where $\rho$ denotes spectral radius of given matrix. Newton's iteration with initial matrix $V_{0}$ satisfying Schulz's condition is also known as Schulz iterative method. Ben-Israel with his collaborators have modified this iterative method (see [2,3] and [4]) to find Moore-Penrose inverse of given rectangular matrix with suitable initial matrices. In the recent years, many have attempted to improvise these iterative methods to find MoorePenrose inverse, Drazin inverse and outer inverses with specific column space and row space. A few examples in this regard are work by Stanimirović with his collaborators (see $[12,13,16,17])$ and others (see, $[5,6,11,18,19])$. In this article, inspired by Stanimirović's work, we consider core-EP inverse as a special case of an outer inverse to propose an iterative method and discuss its convergence.

\section{Preliminaries}

The class of all $m \times n$ matrices over complex field is denoted by $\mathbb{C}^{m \times n}$. Given a matrix $A$, we denote the conjugate transpose, column space, row space, null space, rank and index of the matrix by $A^{*}, \mathcal{C}(A), \mathcal{R}(A), \mathcal{N}(A), \operatorname{rank}(A)$ and $\operatorname{ind}(A)$, respectively. Consider the Penrose conditions:

$$
\begin{array}{llll}
\text { (1) } & A X A=A & (2) & X A X=X \\
(3) & (A X)^{*}=A X & (4) & (X A)^{*}=X A
\end{array}
$$

If $A$ is a square matrix, in addition to the above Penrose conditions we consider the following:

$$
\text { (5) } A X=X A \quad\left(1^{k}\right) \quad X A^{k+1}=A^{k} \quad\left({ }^{k} 1\right) \quad A^{k+1} X=A^{k} \text {, }
$$

where $k$ is some positive integer.

A matrix $G$ satisfying Penrose conditions $(1)-(4)$ is called the Moore-Penrose inverse, satisfying condition (1) is called a generalized inverse, satisfying condition (2) is called an outer inverse, satisfying the conditions (1), (2) and (5) is called the group inverse, satisfying $(2),(5)$ and $\left(1^{k}\right)$ is called Drazin inverse. In general, a matrix $G$ satisfying the conditions $i, j, \ldots, k$ is called $\{i, j, \ldots, k\}$-inverse.

An arbitrary generalized inverse is denoted by $A^{-}$, an outer inverse by $A^{=}$, reflexive generalized inverse satisfying conditions (1) and (2) by $A_{r}^{-}$, the Moore-Penrose inverse by $A^{\dagger}$, the group inverse by $A^{\#}$ and the Drazin inverse by $A^{D}$.

There exists class of generalized inverses and outer inverses not among any of $\{i, j, \ldots, k\}$-inverses and the core-EP and *core-EP inverse are among them.

Definition 2.1 (CORE-EP InVERse, [9]). A matrix $G$ is said to be core-EP inverse of the square matrix $A$ if $G$ is an outer inverse of $A$ with

$$
\mathcal{C}(G)=\mathcal{R}(G)=\mathcal{C}\left(A^{d}\right),
$$

where $d$ is the index of $A$. Similarly, ${ }^{*}$ core-EP inverse is defined by replacing $\mathcal{C}\left(A^{d}\right)$ by $\mathcal{R}\left(A^{d}\right)$ in the above definition. 
We use $A^{\oplus}$ to denote the core-EP inverse of $A$.

The existence of $A \oplus$ is proved by Manjunatha Prasad et al., in [9] and some of the properties are also discussed with the help of the understanding that core-EP inverse of $A$, whenever it exists, is an outer inverse of $A$ with its row and column spaces are identical, and it is equals to the column space of $A^{d}$. We use the result of the following theorem in our work extensively.

Whenever the index of $A$ is 1 , the core-EP inverse is called as core-EP generalized inverse of $A$. Note that, whenever core-EP generalized inverse exists, it is a generalized inverse whose column space and row space are identical to the column space of $A$.

Theorem 2.2 (TheOREM 3.5, [9]). Let $A$ be a square matrix of index $d$. Then the following statements are equivalent.

(i) Core-EP inverse of $A$ exists

(ii) $\operatorname{rank}\left(A^{* d} A^{d+1}\right)=\operatorname{rank}\left(A^{d}\right)$.

Further, whenever core-EP inverse exists, it is unique and given by

$$
A^{d}\left(A^{* d} A^{d+1}\right)^{-} A^{* d} .
$$

Note that, for a matrix $A$ over real or complex field, it is always true that $\operatorname{rank}\left(A^{* d} A^{d+1}\right)=$ $\operatorname{rank}\left(A^{d}\right)$ whenever $d$ is the index of $A$. Hence, the existence and uniqueness of the core-EP inverse of a square matrix over real or complex field. Whenever the index of $A$ is one, the core-EP inverse is $\left\{2,3,1^{k}\right\}$-inverse with $k=1$, in which case the core-EP inverse is known as core-EP generalized inverse.

Apart from the expression given in (2.1) the core-EP inverse can be obtained by different methods and the bordering method is one among them. In [10], the authors have given the bordering method to obtain core-EP inverse of a square matrix. In the present article, we continue to discuss an iterative method, following Stanimirović and others work, to compute core-EP inverse.

\section{Iterative Method and its Convergence}

In this section, we consider recursive formula as suggested by Petković-Stanimirović in [12] to find Moore-Penrose inverse and use the same to find core-EP inverse. For the benefit of readers we describe the origin of the recursion formula in detail.

Throughout this section let $A$ be a square matrix of size $n$ with index $d$ and let $X$ be the core-EP inverse of $A$. Since $X$ is an outer inverse and statisfies $X=X A X$, we have for any real number $\beta$,

$$
X=X-\beta(X A X-X)=(1+\beta) X-\beta X A X .
$$


Hence, we propose the recursive relation given by

$$
X_{k+1}=(1+\beta) X_{k}-\beta X_{k} A X_{k}
$$

If the sequence of recursive matrices $X_{k}$ converges to $Y$ (say) then $Y$ is an outer inverse of $A$. Noting that $\operatorname{rank}\left(A^{d} A^{* d+1}\right)=\operatorname{rank}\left(A^{d}\right)$ and $d$ the index of $A$, we have that

$$
\mathcal{C}\left(A^{d} A^{* d+1}\right)=\mathcal{C}\left(A^{d}\right) \text { and } \mathcal{R}\left(A^{d} A^{* d+1}\right)=\mathcal{R}\left(A^{* d+1}\right)=\mathcal{C}\left(A^{d}\right) .
$$

So, we propose the initial matrix $X_{0}$ given by

$$
X_{0}=\alpha A^{d} A^{* d+1} \text { for some nonzero real } \alpha .
$$

Now, from the definition of $X_{0}, X_{k}$ and the core-EP inverse we have that

$$
X A X_{k}=X_{k}=X_{k} A X \text { for all } k=1,2, \ldots
$$

Let us denote $X_{k}-X$, the error matrices by $E_{k}$. It is also clear that $A X$ and $A X_{k}$ commutes since $(A X)\left(A X_{k}\right)=A X_{k}=\left(A X_{k}\right)(A X)$. In the following theorem we provide a necessary condition for the convergence of iterative with recursive formula (3.1) with intial matrix as given in (3.2).

THEOREM 3.1. The recursive matrix $X_{k}$ as given in (3.1) with the initial matrix (3.2) converges to the core-EP inverse $X$ if

$$
A X_{0}-A X<1, \quad 0<\beta \leq 1
$$

for any matrix multiplicative norm . .

Proof. Let - be a matrix multiplicative norm such that

$$
A X_{0}-A X<1, \quad 0<\beta \leq 1
$$

From the definitions of $X_{0}, X_{k}$ and the core-EP inverse we have that

$$
E_{k+1}=X_{k+1}-X=X A X_{k+1}-X A X \leq X A E_{k+1} .
$$

Further,

$$
\begin{aligned}
A E_{k+1}=A X_{k+1}-A X & =(1+\beta) A X_{k}-\beta A X_{k} A X_{k} . \\
& =A X_{k}+\beta A X_{k} A X-\beta A X_{k} A X_{k}-A X \\
& =-\left(\beta A X_{k}-A X\right)\left(A X_{k}-A X\right) \\
& =\left[-\beta\left(A X_{k}-A X\right)+\beta A X-A X\right]\left(A X_{k}-A X\right) \\
& =\beta\left(A X_{k}-A X\right)^{2}+(1-\beta)\left(A X_{k}-A X\right) \\
& =-\beta\left(A E_{k}\right)^{2}+(1-\beta) A E_{k} .
\end{aligned}
$$


Therefore,

$$
A E_{k+1} \leq \beta\left\|\left(A E_{k}\right)^{2}\right\|+(1-\beta) A E_{k}
$$

Since - is a matrix multiplicative norm, from (3.6) we get that

$$
A E_{k+1} \leq A E_{k}\left(\beta A E_{k}+(1-\beta)\right) \text {. }
$$

Given that $A E_{0}<1$. From (3.7) we get, $A E_{k}<1, \beta A E_{k}+(1-\beta)<1$ and therefore,

$$
A E_{k+1}<A E_{k} .
$$

In other words, $A E_{k}$ is a strictly monotonically decreasing sequence of positive real numbers. Hence, $A E_{k} \rightarrow e$ (say), where $0 \leq e<1$. Now referring to (3.7), we get that $e=0$.

In other words, $A E_{k} \rightarrow 0$. Now from (3.4) we get that, $E_{k} \rightarrow 0$, which proves the theorem.

Note that the convergence of the iterative method was discussed in the above theorem under the condition $\left\|A\left(\alpha A^{d} A^{* d+1}-X\right)\right\|<1$. Now we will proceed to identify an $\alpha$ satisfying this condition. First we recall a usefull result related to matrix norm from the literature. For the details, we refer to $[1,7]$.

LeMmA 3.2. Let $A$ be any matrix over complex field and $\epsilon>0$ be given. Then there exists a matrix multiplicative norm · such that

$$
\rho(A) \leq A \leq \rho(A)+\epsilon
$$

In view of Lemma 3.2, the proposed iterative method converges if

$$
\rho\left(A\left(\alpha A^{d} A^{* d+1}-X\right)\right)<1
$$

for an approriate choice of $\alpha$. Now, choose

$$
\alpha=\frac{1}{2 \operatorname{tr}\left(A^{d+1} A^{* d+1}\right)},
$$

where $\operatorname{tr}(\cdot)$ refers to the trace of $(\cdot)$. Since $A^{d+1} A^{* d+1}$ is positive semi-definite matrix, we have that

$$
\alpha<\frac{1}{\rho\left(A^{d+1} A^{* d+1}\right)} .
$$

For any nonzero eigenvector $x$ of $A^{d+1} A^{* d+1}$ corresponding to an eigenvalue $\gamma$, note that $A X_{0} x=\alpha \gamma x$ and therefore,

$$
\left(A X-A X_{0}\right) x=(1-\alpha \gamma) x
$$


as $A X$ is an orthogonal projection onto $\mathcal{C}\left(A^{d}\right)$. Since $0 \leq \gamma \leq \rho\left(A^{d+1} A^{* d+1}\right)$, we have that $0 \leq \alpha \gamma<1$ and therefore $1-\alpha \gamma<1$. This in turn implies that

$$
\rho\left(A X-A X_{0}\right)<1 \text {. }
$$

Then we have the following result on converging iterative method to obtain core-EP inverse.

THEOREM 3.3. Let $A$ be a square matrix with index $d$ and $X$ be the core-EP inverse of $A$. For $\beta \in(0,1], \alpha=\frac{1}{2 \operatorname{tr}\left(A^{d+1} A^{* d+1}\right)}$ consider the iteration with recursive equation (3.1) with initial matrix (3.2). Then the following hold.

(i) $\rho\left(A X_{0}-A X\right)<1$.

(ii) The recursive matrix $X_{k}$ converges to $X$.

We have similar iterative method with the initial matrix as

$$
X_{0}=\alpha A^{* d+1} A^{d}
$$

to find * core-EP inverse.

THEOREM 3.4. Let $A$ be a square matrix with index $d$ and $X$ be the ${ }^{*}$ core-EP inverse of $A$. For $\beta \in(0,1], \alpha=\frac{1}{2 \operatorname{tr}\left(A^{* d+1} A^{d+1}\right)}$ consider the iteration with recursive equation (3.1) with initial matrix (3.15). Then the following hold.

(i) $\rho\left(X_{0} A-X A\right)<1$.

(ii) The recursive matrix $X_{k}$ converges to $X$.

\section{Influence of Roundoff Errors}

Inspite of the iterative method proposed in the section 3 converges theoretically, in practice, it may not be reflected in the actual computation using any efficient computing system. It is mainly due to floating point arithmetic used in the program which is compatible with the computing system, and hence roundoff error occurs.

In this section we attempt to analyze the roundoff errors which causes the divergences of the recursive relation (3.1) with (3.2). Consider

$$
X_{k}^{\prime}=X_{k}+\Delta_{k},
$$

where the computed matrix $X_{k}^{\prime}$ differ from the original matrix $X_{k}$ by the error matrix $\Delta_{k}$ in $k$ th iteration.

The following theorem by Petković and Stanimirović [13, Theorem 2.3] also holds for the present iterative method and the proof is similar. 
THEOREM 4.1. Consider the recursive realtion (3.1) with (3.2) and assume that the roundoff error in pth iteration initiates $\mathcal{N}\left(X_{p}^{\prime}\right) \quad \mathcal{N}\left(X_{p}^{\prime} A X_{p}^{\prime}\right)$. Then the resulting method

$$
X_{k+1}^{\prime}=(1+\beta) X_{k}^{\prime}-\beta X_{k}^{\prime} A X_{k}^{\prime}, \quad k \geq p,
$$

diverges and $X_{k}^{\prime} \geq c \cdot(1+\beta)^{k-p}$, where $c>0$ is a constant which depends only on $X_{p}^{\prime}$.

\section{Complexity Analysis}

Irrespective of efficiency of the given computing system, it is manditory to define a stoping criteria to obtain an optimal result in computation. The result given in the following theorem describes the quality of convergence of recursive matrices. The result is from [12, Theorem 3.4] valid in the present context also and so we present the same without proof.

THEOREM 5.1. Suppose the recursive relation (3.1) with the initial matrix (3.2) converges, then

$$
\lim _{k \rightarrow \infty} \frac{t_{k+1}}{t_{k}}=\lim _{k \rightarrow \infty} \frac{s_{k+1}}{s_{k}}=\lim _{k \rightarrow \infty} \frac{d_{k+1}}{d_{k}}=1-\beta
$$

where $t_{k}=A E_{k}, s_{k}=E_{k}$ and $d_{k}=X_{k+1}-X_{k}$.

We propose the following two possible stopping criteria, where the second is derived from (5.1).

(a) For a given tolerance $\epsilon>0$, we stop the algorithm if

$$
d_{k}=X_{k+1}-X_{k}<\epsilon
$$

(b) For a given tolerance $\epsilon>0$, we stop the algorithm if

$$
\left|\frac{d_{k+1}}{d_{k}}-(1-\beta)\right|<\epsilon .
$$

Algorithm 1 gives the pseudocode for a function COREEPINVITER that computes the approximate core-EP inverse of a square matrix $A$ of index $d$ using a given value of $\beta$ and tolerance $\epsilon$.

From Theorem 5.1, $\lim _{k \rightarrow \infty} \frac{d_{k}}{d_{k-1}}=1-\beta$. Thus for sufficiently large $k, d_{k} \approx(1-$ $\beta) d_{k-1}$, which suggests that for some large $k_{0}$ and for all $k \geq k_{0}, d_{k} \approx(1-\beta)^{k-k_{0}} d_{k_{0}}$.

This allows us to compute the minimum number of iterations $k$ required to reduce the residual norm $d_{k}$ to the given tolerance.

LEMmA 5.2. Let $A$ be a square matrix. Then there exists a positive integer $k_{0}$ such that for all $\epsilon>0$, the minimum number of iterations $k$ taken by Algorithm 1 to compute 


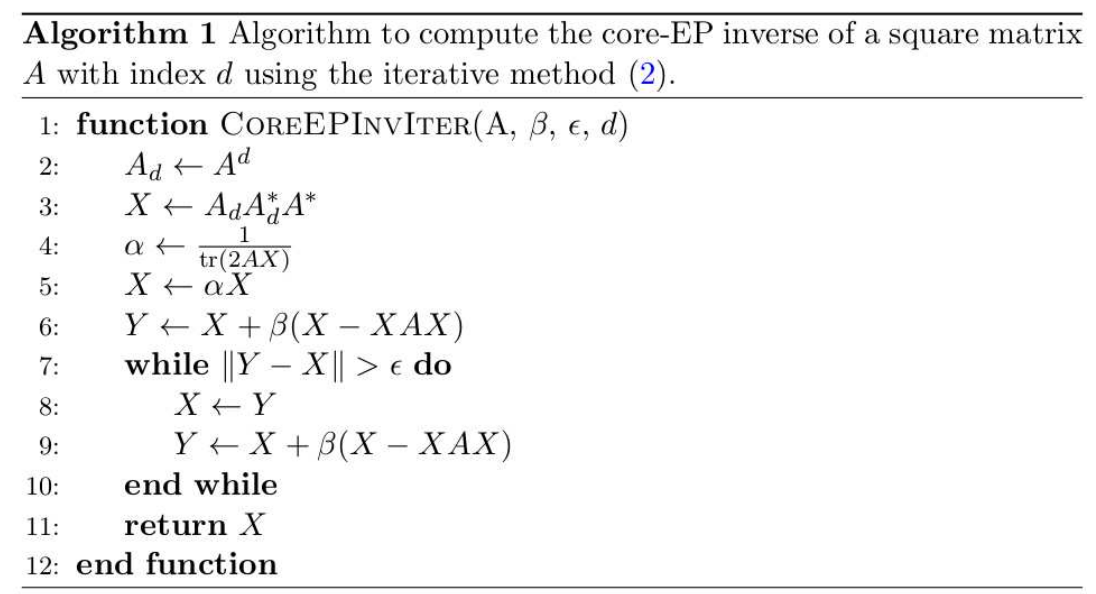

Figure 1

an approximate core-EP inverse $X_{k}$ of $A$ with residual norm $d_{k}=X_{k+1}-X_{k} \leq \epsilon$ is

$$
k=\left\lceil d_{k_{0}}+\frac{\log \left(\epsilon / d_{k_{0}}\right)}{\log (1-\beta)}\right\rceil,
$$

where $\lceil\cdot\rceil$ denotes the ceiling function.

Proof. We define a sequence $\delta_{k}=\frac{d_{k}}{d_{k-1}}-(1-\beta)$ and observe that by Theorem 5.1, $\delta_{k} \rightarrow 0$ as $k \rightarrow \infty$. Thus, for all $\gamma>0$, there exists a positive integer $k_{\gamma}$ such that $\forall k \geq k_{\gamma}$

$$
\left|\delta_{k}\right|=\left|\frac{d_{k}}{d_{k-1}}-(1-\beta)\right| \leq \gamma
$$

Then

$$
d_{k} \geq(1-\beta-\gamma) d_{k-1}
$$

Since this holds for all $k \geq k_{\gamma}$, we may replace $d_{k-1}$ by $(1-\beta-\gamma) d_{k-2}$ without affecting the inequality. Proceeding similarly, we see that

$$
d_{k} \geq(1-\beta-\gamma)^{k-k_{\gamma}} d_{k_{\gamma}}
$$

Then for $d_{k} \leq \epsilon$, we must have

$$
(1-\beta-\gamma)^{k-k_{\gamma}} d_{k_{\gamma}} \leq \epsilon,
$$

which implies that for all $\gamma \in(0,1-\beta)$,

$$
k-k_{\gamma} \geq \frac{\log \left(\epsilon / d_{k_{\gamma}}\right)}{\log (1-\beta-\gamma)} .
$$


Note that $\log \epsilon / d_{k_{\gamma}}$ and $\log (1-\beta-\gamma)$ are negative. Now, allowing $\gamma$ to approach 0 , we obtain

$$
k \geq k_{0}+\frac{\log \left(\epsilon / d_{k_{0}}\right)}{\log (1-\beta)}
$$

for some $k_{0}$. Clearly, the minimum possible integer value of $k$ satisfying this inequality is $\left\lceil k_{0}+\frac{\log \left(\epsilon / d_{k_{\gamma}}\right)}{\log (1-\beta)}\right\rceil$, which concludes the proof.

From Lemma 5.2, it is easy to compute the time complexity of Algorithm 1 and the same is given in the following theorem.

THEOREM 5.3. Let $A$ be a square matrix of order $n$. Then the time complexity of Algorithm 1 is $O\left(n^{p k}\right)$, where

$$
k=\frac{\log \left(\epsilon / d_{k_{0}}\right)}{\log (1-\beta)}
$$

when all matrix-matrix multiplications are performed using a multiplication algorithm of polynomial time complexity $O\left(n^{p}\right)$.

At the time of writing of this paper, matrix-matrix multiplication algorithms of time complexity $O\left(n^{2.373}\right)$ are available [6].

\section{Numerical Examples}

In this section, we present the results of some test of our method using SageMath 8.1 [14].

\subsection{Using fixed number of iterations}

We compare the numerical stability of Algorithm 1 for 1000 iterations with 87 bits (roughly 25 decimals) and 170 bits (roughly 50 decimals) of precision for a matrix whose core-EP inverse is known. The implementation details are given in Appendix 6.2, Listing 1.

EXAMPLE 6.1. Let

$$
A=\left[\begin{array}{ccccccccc}
-36 & -30 & 21 & -18 & 51 & -6 & -60 & 0 & 12 \\
-198 & -228 & 186 & -90 & 378 & -42 & -444 & 48 & -12 \\
238 & 252 & -190 & 112 & -404 & 46 & 468 & -40 & -12 \\
-186 & -216 & 174 & -84 & 360 & -42 & -420 & 48 & -12 \\
226 & 240 & -184 & 100 & -374 & 46 & 444 & -40 & -12 \\
450 & 486 & -372 & 204 & -768 & 90 & 900 & -90 & -18 \\
406 & 438 & -340 & 184 & -704 & 82 & 828 & -76 & -12 \\
-42 & -60 & 60 & -24 & 108 & -18 & -126 & 18 & -18 \\
-206 & -216 & 164 & -92 & 346 & -38 & -408 & 38 & 18
\end{array}\right]
$$


Note that $A$ has index 5 and the core-EP inverse of $A$ is

$$
G=\frac{1}{2880}\left[\begin{array}{ccccccccc}
40 & 10 & -40 & 10 & 10 & 10 & -40 & -40 & 40 \\
-4 & 65 & 34 & 65 & 5 & 5 & -26 & 34 & 26 \\
-52 & 5 & 82 & 5 & -55 & -55 & 22 & 82 & -22 \\
-4 & 65 & 34 & 65 & 5 & 5 & -26 & 34 & 26 \\
20 & 35 & -50 & 35 & 95 & 95 & 10 & -50 & -10 \\
20 & 35 & -50 & 35 & 95 & 95 & 10 & -50 & -10 \\
-28 & -25 & -2 & -25 & 35 & 35 & 58 & -2 & -58 \\
-52 & 5 & 82 & 5 & -55 & -55 & 22 & 82 & -22 \\
28 & 25 & 2 & 25 & -35 & -35 & -58 & 2 & 58
\end{array}\right] .
$$

We have used Algorithm 1 with $\beta=0.05$ to compute the residual norms $t_{k}=A E_{k}{ }_{2}=$ $A\left(X_{k}-X\right)_{2}, s_{k}=E_{k}{ }_{2}=X_{k}-X_{2}$, and $d_{k}=X_{k+1}-X_{k}$ for every ten iterations up to 1000 iterations and plotted these values against the number of iterations in lin-log scale (see Figure 2).
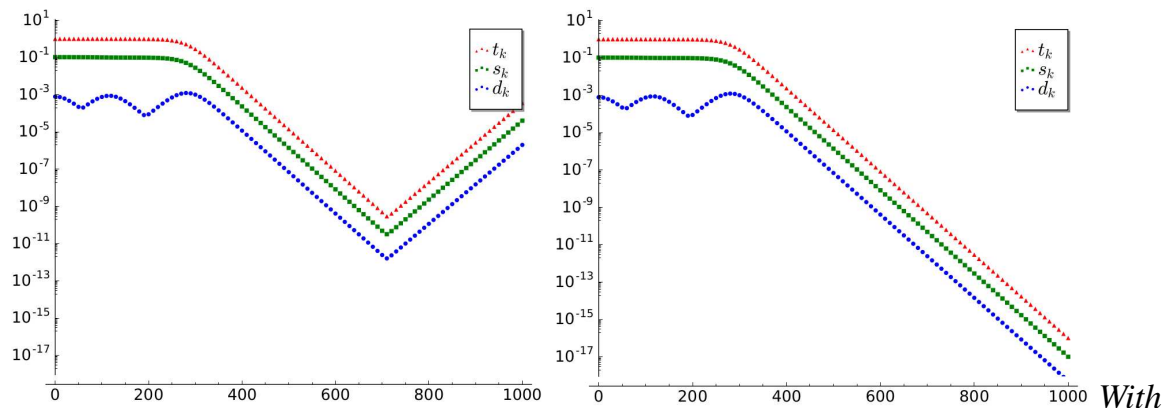

87 bits (roughly 25 decimals) precision With 170 bits (roughly 50 decimals) precision

Figure 2: Residual norms $t_{k}=A E_{k}{ }_{2}, s_{k}=E_{k}{ }_{2}$, and $d_{k}=X_{k+1}-X_{k} 2$ against number of iterations in lin-log scale.

It can be clearly seen from these graphs that the algorithm starts to converge after a few initial steps, following which all three residual norms decrease exponentially over several iterations. This continues until divergence occurs due to roundoff errors. We observe that when we increase the value of the precision from 87 bits to 170 bits, divergence does not occur within the first 1000 iterations, which results in a better approximation of the core-EP inverse.

\subsection{Using stopping criteria}

Now we analyse the performance of Algorithm 1 for several randomly generated matrices with different indices. The stopping criteria (5.2) and (5.3) are implemented in Listings 2 and 3 and the results are shown in Tables 1 and 2 respectively. Since we use floating point arithmetic, the algorithm may diverge before these criteria are met. 
Therefore, it is also proposed to set a (large) upper bound on the number of iterations as an additional stopping criterion.

Table 1: Performance of Algorithm 1 with stopping criterion (5.2). The values shown are the number of iterations (Iter.) required by the algorithm to compute the core-EP inverse of a matrix of order $n$ and index $d$, with the residual norm $\left(d_{k}\right)$ below the specified tolerance $\epsilon$ for different values of $\beta$.

\begin{tabular}{|c|c|c|c|c|c|c|c|c|c|c|c|c|c|c|c|}
\hline & \multirow{3}{*}{$\beta$} & \multicolumn{4}{|c|}{0.15} & \multicolumn{6}{|c|}{0.2} & \multicolumn{4}{|c|}{0.25} \\
\hline & & \multicolumn{2}{|c|}{$1.0 \times 10^{-10}$} & \multicolumn{2}{|c|}{$1.0 \times 10^{-20}$} & \multicolumn{2}{|c|}{$1.0 \times 10^{-20}$} & \multicolumn{2}{|c|}{$1.0 \times 10^{-30}$} & \multicolumn{2}{|c|}{$1.0 \times 10^{-50}$} & \multicolumn{2}{|c|}{$1.0 \times 10^{-80}$} & \multicolumn{2}{|c|}{$1.0 \times 10^{-100}$} \\
\hline & & Iter. & $d_{k}$ & er. & $d_{k}$ & Iter. & $d_{k}$ & Iter. & $d_{k}$ & Iter. & $d_{k}$ & Iter. & $d_{k}$ & Iter. & $d_{k}$ \\
\hline \multirow{3}{*}{10} & 3 & 213 & $3 \times 1$ & 355 & $\times 10$ & 2 & $x 1$ & 105 & $8.4 \times$ & 11 & $\times 10$ & 241 & $4 \times 10^{-81}$ & 401 & \\
\hline & 6 & 262 & $9 \times 10^{-11}$ & 403 & $9.9 \times 10^{-2}$ & 2 & $9.0 \times 10^{-21}$ & 105 & $9.4 \times 10^{-31}$ & 312 & $8.1 \times 10^{-51}$ & 241 & $8.4 \times 10^{-81}$ & 401 & $8.6 \times 10^{-101}$ \\
\hline & 9 & 128 & $9.2 \times 10^{-11}$ & 270 & $8.7 \times 10^{-21}$ & 1 & $9.9 \times 10^{-21}$ & 105 & $8.2 \times 10^{-31}$ & 311 & $9.0 \times 10^{-51}$ & 241 & $9.3 \times 10^{-81}$ & 401 & $.5 \times 10^{-101}$ \\
\hline \multirow{3}{*}{20} & 8 & 339 & $4 \times 10^{-11}$ & 481 & $8.9 \times 1$ & 2 & -21 & 105 & -31 & 311 & -51 & 241 & $9.5 \times 10^{-81}$ & 401 & $7 \times 10^{-101}$ \\
\hline & 12 & 397 & $9.7 \times 10^{-11}$ & 539 & $9.3 \times 10^{-21}$ & 2 & $8.4 \times 10^{-21}$ & 105 & $8.7 \times 10^{-31}$ & 311 & $9.5 \times 10^{-51}$ & 241 & $9.8 \times 10^{-81}$ & 402 & $7.5 \times 10^{-101}$ \\
\hline & 16 & 383 & $8.6 \times 10^{-11}$ & 524 & $9.6 \times 10^{-21}$ & 2 & $8.7 \times 10^{-21}$ & 105 & $9.1 \times 10^{-31}$ & 311 & $9.9 \times 10^{-51}$ & 242 & $7.7 \times 10^{-81}$ & 402 & $7.9 \times 10^{-101}$ \\
\hline \multirow{3}{*}{50} & 20 & 520 & $8 \times 10^{-11}$ & 661 & $9.8 \times 10^{-2}$ & 2 & $8.9 \times 10^{-21}$ & 105 & $9.3 \times 10^{-31}$ & 312 & $8.1 \times 10^{-51}$ & 241 & $8.4 \times 10^{-81}$ & 401 & $.6 \times 10^{-101}$ \\
\hline & 35 & 391 & $8.6 \times 10^{-11}$ & 890 & $9.3 \times 10^{-21}$ & 2 & $8.4 \times 10^{-21}$ & 105 & $8.8 \times 10^{-31}$ & 311 & $9.6 \times 10^{-51}$ & 241 & $9.9 \times 10^{-81}$ & 402 & $7.6 \times 10^{-101}$ \\
\hline & 45 & 165 & $8.6 \times 10^{-11}$ & 306 & $9.6 \times 10^{-21}$ & 2 & $8.7 \times 10^{-21}$ & 105 & $9.1 \times 10^{-31}$ & 311 & $9.9 \times 10^{-51}$ & 242 & $7.7 \times 10^{-81}$ & 402 & $7.8 \times 10^{-101}$ \\
\hline \multirow{3}{*}{75} & 3 & 371 & $8.7 \times 10^{-11}$ & 828 & $9.9 \times 10^{-21}$ & 2 & $9.0 \times 10^{-21}$ & 105 & $9.4 \times 10^{-31}$ & 312 & $8.2 \times 10^{-51}$ & 241 & $8.5 \times 10^{-81}$ & 401 & $8.7 \times 10^{-101}$ \\
\hline & 50 & 196 & $9.1 \times 10^{-11}$ & 1158 & $9.0 \times 10^{-21}$ & 2 & $8.1 \times 10^{-21}$ & 105 & $8.5 \times 10^{-31}$ & 311 & $9.2 \times 10^{-51}$ & 241 & $9.6 \times 10^{-81}$ & 401 & $9.8 \times 10^{-101}$ \\
\hline & 70 & 161 & $9.2 \times 10^{-11}$ & 303 & $8.7 \times 10^{-21}$ & 1 & $9.9 \times 10^{-21}$ & 105 & $8.2 \times 10^{-31}$ & 311 & $8.9 \times 10^{-51}$ & 241 & $9.3 \times 10^{-81}$ & 401 & $9.5 \times 10^{-101}$ \\
\hline \multirow{3}{*}{100} & te & 459 & $9.4 \times 10^{-11}$ & 1016 & $8.6 \times 10^{-21}$ & 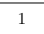 & $9.8 \times 10^{-21}$ & 105 & $8.2 \times 10^{-31}$ & 311 & $8.9 \times 10^{-51}$ & 241 & $9.2 \times 10^{-81}$ & 101 & $4 \times 10^{-101}$ \\
\hline & 81 & 165 & $8.6 \times 10^{-11}$ & 778 & $8.8 \times 10^{-21}$ & 1 & $1.0 \times 10^{-20}$ & 719 & $9.4 \times 10^{-31}$ & 926 & $8.2 \times 10^{-51}$ & 241 & $8.5 \times 10^{-81}$ & 401 & $8.6 \times 10^{-101}$ \\
\hline & 90 & 149 & $8.8 \times 10^{-11}$ & 825 & $9.6 \times 10^{-21}$ & 2 & $8.7 \times 10^{-21}$ & 105 & $9.1 \times 10^{-31}$ & 998 & $8.8 \times 10^{-51}$ & 241 & $9.1 \times 10^{-81}$ & 401 & $9.3 \times 10^{-101}$ \\
\hline
\end{tabular}

Table 2: Performance of Algorithm 1 with stopping criterion (5.3). The values shown are the number of iterations (Iter.) required by the algorithm to compute the core-EP inverse of a matrix of order $n$ and index $d$, with $\left|\frac{d_{k+1}}{d_{k}}-(1-\beta)\right|$ below the specified tolerance $\epsilon$ for different values of $\beta$.

\begin{tabular}{|c|c|c|c|c|c|c|c|c|c|c|c|c|c|c|c|}
\hline & \multirow{3}{*}{${ }_{d} \beta$} & \multicolumn{4}{|c|}{0.25} & \multicolumn{6}{|c|}{0.5} & \multicolumn{4}{|c|}{0.75} \\
\hline & & \multicolumn{2}{|c|}{$1.0 \times 10^{-10}$} & \multicolumn{2}{|c|}{$1.0 \times 10^{-20}$} & \multicolumn{2}{|c|}{$1.0 \times 10^{-20}$} & \multicolumn{2}{|c|}{$1.0 \times 10^{-30}$} & \multicolumn{2}{|c|}{$1.0 \times 10^{-50}$} & \multicolumn{2}{|c|}{$1.0 \times 10^{-80}$} & \multicolumn{2}{|c|}{$1.0 \times 10^{-100}$} \\
\hline & & Iter. & $d_{k}$ & er. & $d_{k}$ & Iter. & $d_{k}$ & Iter. & $d_{k}$ & Iter. & $d_{k}$ & ter. & $d_{k}$ & Iter. & $d_{k}$ \\
\hline \multirow{3}{*}{10} & 5 & 163 & $5 \times 10$ & 166 & $\times 10$ & 72 & $1.5 \times 10$ & 73 & $7.6 \times 10^{-18}$ & 74 & $3.8 \times 10^{-}$ & 42 & $2.3 \times 10^{-18}$ & 43 & $5.7 \times 1$ \\
\hline & 7 & 177 & $8.2 \times 10^{-17}$ & 79 & $4.6 \times 10^{-17}$ & 86 & $4.6 \times 10^{-18}$ & 87 & $2.3 \times 10^{-18}$ & 88 & $1.1 \times 10^{-18}$ & 50 & $6.0 \times 10^{-18}$ & 51 & $1.5 \times 10^{-18}$ \\
\hline & 9 & 13 & $5.9 \times 10^{-18}$ & 131 & $4.5 \times 10^{-18}$ & 55 & $8.5 \times 10^{-18}$ & 56 & $4.2 \times 10^{-18}$ & 57 & $2.1 \times 10^{-18}$ & 29 & $3.8 \times 10^{-18}$ & 30 & $9.5 \times 10^{-19}$ \\
\hline \multirow{3}{*}{20} & 10 & & 10 & 25 & 10 & 125 & $(10-17$ & 127 & $\times 10^{-18}$ & 28 & $\times 10^{-18}$ & 79 & $\times 10^{-17}$ & 80 & $\times 10^{-18}$ \\
\hline & 14 & 288 & $.2 \times 10^{-17}$ & 291 & $2.6 \times 10^{-17}$ & 145 & $1.3 \times 10^{-17}$ & 147 & $3.2 \times 10^{-18}$ & 148 & $1.6 \times 10^{-18}$ & 94 & $4.4 \times 10^{-18}$ & 95 & $1.1 \times 10^{-18}$ \\
\hline & 18 & 125 & $5 \times 10^{-17}$ & 130 & $5.9 \times 10^{-18}$ & 56 & $4.2 \times 10^{-18}$ & 57 & $2.1 \times 10^{-18}$ & 58 & $1.1 \times 10^{-18}$ & 30 & $9.5 \times 10^{-19}$ & 31 & $2.4 \times 10^{-19}$ \\
\hline \multirow{3}{*}{50} & 20 & 37 & $9 \times 10^{-17}$ & 37 & $2.2 \times 10^{-17}$ & 190 & $.3 \times 10^{-17}$ & 191 & $6.4 \times 10^{-18}$ & 192 & $3.2 \times 10^{-18}$ & 125 & $3.0 \times 10^{-17}$ & 126 & $7.6 \times 10^{-18}$ \\
\hline & 35 & 504 & $4.5 \times 10^{-17}$ & 508 & $1.4 \times 10^{-17}$ & 263 & $1.5 \times 10^{-17}$ & 264 & $7.4 \times 10^{-18}$ & 265 & $3.7 \times 10^{-18}$ & 179 & $9.8 \times 10^{-18}$ & 180 & $2.5 \times 10^{-18}$ \\
\hline & 45 & 298 & $2.7 \times 10^{-17}$ & 302 & $8.7 \times 10^{-18}$ & 151 & $4.0 \times 10^{-18}$ & 152 & $2.0 \times 10^{-18}$ & 153 & $1.0 \times 10^{-18}$ & 97 & $7.8 \times 10^{-18}$ & 98 & $1.9 \times 10^{-18}$ \\
\hline \multirow{3}{*}{75} & 30 & 48 & $\times 10^{-17}$ & 481 & $4.4 \times 10^{-17}$ & 251 & $7.6 \times 10^{-18}$ & 252 & $3.8 \times 10^{-18}$ & 253 & $1.9 \times 10^{-18}$ & 169 & $1.8 \times 10^{-17}$ & 170 & $4.5 \times 10^{-18}$ \\
\hline & 50 & 661 & $5.4 \times 10^{-17}$ & 662 & $4.1 \times 10^{-17}$ & 349 & $2.5 \times 10^{-17}$ & 351 & $6.2 \times 10^{-18}$ & 352 & $3.1 \times 10^{-18}$ & 242 & $8.1 \times 10^{-18}$ & 243 & $2.0 \times 10^{-18}$ \\
\hline & 70 & 197 & $6.9 \times 10^{-18}$ & 198 & $5.2 \times 10^{-18}$ & 92 & $7.0 \times 10^{-18}$ & 94 & $1.8 \times 10^{-18}$ & 95 & $8.8 \times 10^{-19}$ & 55 & $6.2 \times 10^{-18}$ & 56 & $1.5 \times 10^{-18}$ \\
\hline & 50 & 673 & $9.4 \times 10^{-17}$ & 679 & $1.7 \times 10^{-17}$ & 359 & $4.3 \times 10^{-18}$ & 361 & $1.1 \times 10^{-18}$ & 362 & $5.3 \times 10^{-19}$ & 247 & $1.2 \times 10^{-17}$ & 248 & $3.0 \times 10^{-18}$ \\
\hline & 80 & 455 & $2.3 \times 10^{-17}$ & 459 & $7.3 \times 10^{-18}$ & 236 & $10^{-18}$ & 237 & $4.0 \times 10^{-18}$ & 238 & 18 & 159 & $1 \times 10^{-17}$ & 160 & $2.7 \times 10^{-}$ \\
\hline & 90 & 498 & $2.1 \times 10^{-17}$ & 499 & $1.6 \times 10^{-17}$ & 260 & $5.9 \times 10^{-18}$ & 261 & $3.0 \times 10^{-18}$ & 263 & $7.4 \times 10^{-19}$ & 43 & $3.3 \times 10^{-18}$ & 44 & $8.3 \times 10^{-19}$ \\
\hline
\end{tabular}




\section{Appendix: Implementation Details}

Listing 1: SageMath function to compute the core-EP inverse of a square matrix $A$ using the iterative method (3.1) with fixed number of iterations.

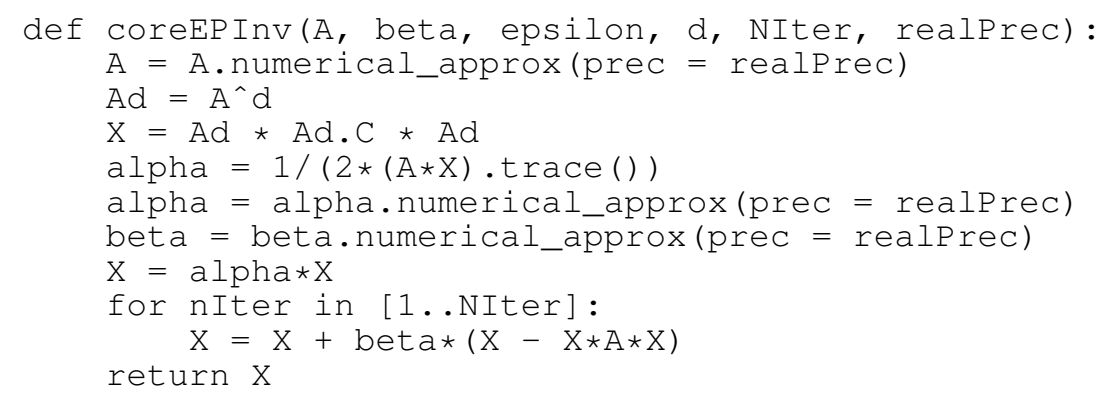

Listing 2: SageMath function to compute the core-EP inverse of a square matrix $A$ using the iterative method (3.1) with stopping criterion (5.2).

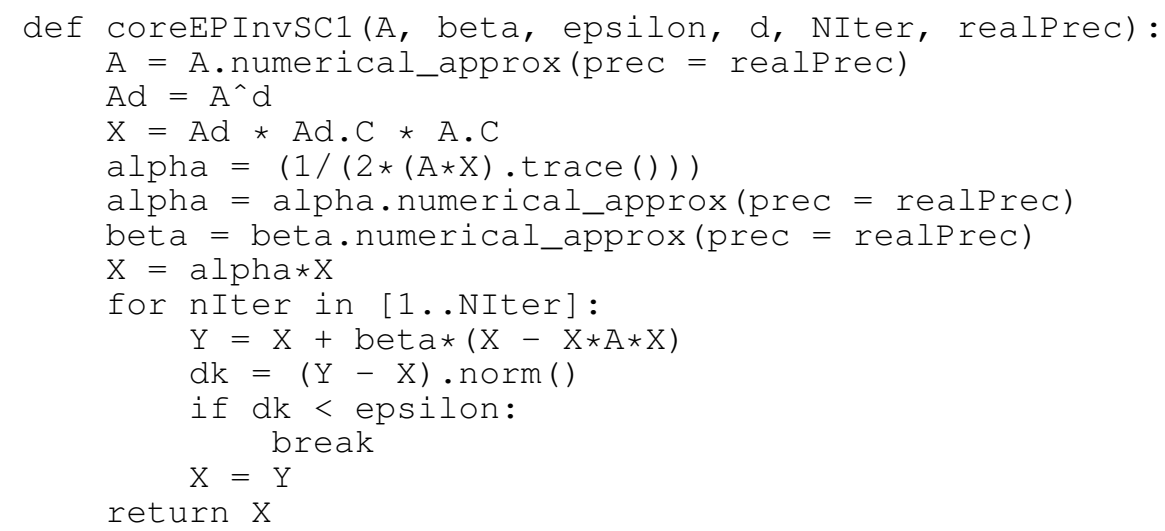

Listing 3: SageMath function to compute the core-EP inverse of a square matrix $A$ using the iterative method (3.1) with stopping criterion (5.3).

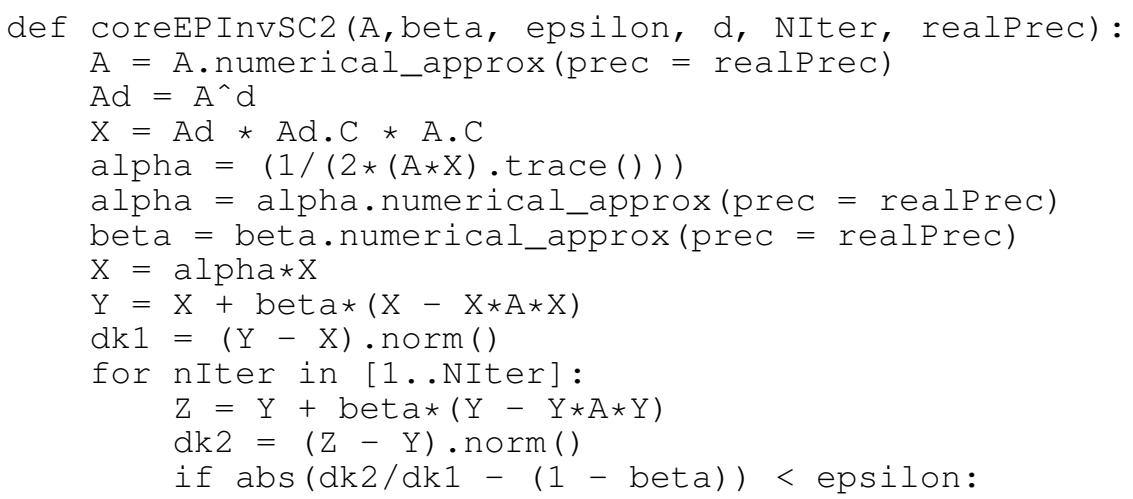




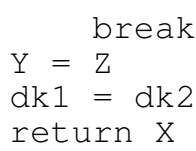

\section{Acknowledgement}

The authors(Manjunatha Prasad Karantha and David Raj Micheal) acknowledge the support by Science and Engineering Research Board (DST, Govt. of India) under Extra Mural Research Funding Scheme (SR/S4/MS:870/14).

\section{REFERENCES}

[1] A. Ben-Israel and T.N.E. Greville. Generalized inverses: Theory and Applications. Springer Science \& Business Media. 2003.

[2] A. Ben-Israel. An iterative method for computing the generalized inverse of an arbitrary matrix. Math, Comp. 19: 452-455. 1965.

[3] A. Ben-Israel. A note on an iterative method for generalized inversion of matrices. Math. Comp. 20: 439-440. 1966.

[4] A. Ben-Israel and D. Cohen. On iterative computation of generalized inverses and associated projections. SIAM J. Numer. Anal. 3: 410-419. 1966.

[5] H. Chen and Y. Wang. A family of Higher-Order Convergent Iterative Methods for Computing the Moore-Penrose inverse. Applied Mathematics and Computation. 218(8): 4012-4016. 2011.

[6] A.M. Davie and A.J. Stothers. Improved bound for complexity of matrix multiplication. Proceedings of the Royal Society of Edinburgh: Section A Mathematics 143(2): 351-369. 2013.

[7] A.S. Householder. The theory of matrices in numerical analysis, Blaisdell Publ. Co., New York. 1964.

[8] K. Manjunatha Prasad, K.P.S. Bhaskara Rao and R.B. Bapat. Generalized inverses over integral domains II. Group inverses and Drazin inverses. Linear and Multilinear Algebra. 146: 31-47. 1991.

[9] K. Manjunatha Prasad and K.S. Mohana. Core-EP inverse. Linear and Multilinear Algebra. 62(6): 792-802. 2014.

[10] K. Manjunatha Prasad and David Raj Micheal. Bordering Method to compute Core-EP inverse. Special Matrices. 2018. (Communicated).

[11] X. Liu and Y. Qin. Successive Matrix Squaring Algorithm for Computing the Generalized Inverse $A_{T, S}^{(2)}$. Journal of Applied Mathematics. 2012:12 pages. 2012. Article ID 262034 
[12] M.D. Petković and P.S. Stanimirović. Iterative method for Computing the Moore-Penrose inverse based on Penrose equations. Journal of Computational and applied Mathematics. 235(6): 1604-1613. 2011.

[13] M. D. Petković and P. S. Stanimirović. Two Improvements of the Iterative method for Computing Moore-Penrose inverse based on Penrose Equations. Journal of Computational and Applied Mathematics. 267: 61-71. 2014.

[14] SageMath, the Sage Mathematics Software System (Version 8.1), The Sage Developers. 2017.

[15] G. Schulz. Iterative Berechmmg der reziproken Matrix, Z. Angew. Math. Mech. 13: 57-59. 1933.

[16] F. Soleymani and P. S. Stanimirović. A Higher order Iterative Method for Computing the Drazin inverse. Sci. World J. 2013: 11. 2013.

[17] P. S. Stanimirović, and D.S. Cvetković-Ilić. Successive Matrix Squaring Algorithm for Computing Outer inverses. Applied Mathematics and Computation. 203(1): 19-29. 2008.

[18] Y. Wei. Successive Matrix Squaring Algorithm for Computing the Drazin inverse. Applied Mathematics and Computation. 108(2-3): 67-75. 2000.

[19] L. Weiguo, L. Juan and Q. Tiantian. A Family of Iterative Methods for Computing Moore-Penrose inverse of a Matrix. Linear Algebra and Its Applications. 438(1): 47-56. 2013. 